\title{
Patterns of Relapse Related to High Expression of Ki-67 After Neoadjuvant Chemotherapy in Triple Negative Breast Cancer
}

\author{
Gines Hernandez-Cortes ${ }^{1,}$, Javier Hornedo $^{2}$, Raquel Murillo ${ }^{3}$, Ricardo Sainz De La Cuesta ${ }^{1}$, \\ Lucia Gonzalez-Cortijo ${ }^{2}$
}

${ }^{1}$ Department of Obstetrics and Gynecology, Universitary Hospital Quironsalud Madrid, Madrid, Spain

${ }^{2}$ Department of Medical Oncology, Universitary Hospital Quironsalud Madrid, Madrid, Spain

${ }^{3}$ Department of Pathology, Universitary Hospital Quironsalud Madrid, Madrid, Spain

\author{
Email address: \\ gines.hernandez@quironsalud.es (G. Hernandez-Cortes) \\ ${ }^{*}$ Corresponding author
}

\section{To cite this article:}

Gines Hernandez-Cortes, Javier Hornedo, Raquel Murillo, Ricardo Sainz De La Cuesta, Lucia Gonzalez-Cortijo. Patterns of Relapse Related to High Expression of Ki-67 After Neoadjuvant Chemotherapy in Triple Negative Breast Cancer. Journal of Gynecology and Obstetrics. Vol. 9, No. 5, 2021, pp. 139-144. doi: 10.11648/j.jgo.20210905.11

Received: August 3, 2021; Accepted: August 17, 2021; Published: September 4, 2021

\begin{abstract}
Introduction: Triple negative breast cancer (TNBC) has been classically considered a high-risk subtype. Early TNBC is usually managed with neoadjuvant chemotherapy (NAC). Residual disease after NAC is generally considered a surrogate marker for event free survival in these patients. The aim of this study was to explore the relationship between high $\mathrm{Ki}-67$ expression in residual disease after NAC and disease relapse. Material and methods: This is a retrospective study of 121 patients diagnosed with TNBC, treated with neoadjuvant chemotherapy in our institution between 2008 and 2018. Clinical features, systemic and surgical therapy and pathological response were analyzed. Ki-67 expression was performed in the postNAC surgical specimens when a pathological complete response (pCR) was not achieved. Results were correlated with number and type of relapse, and survival. Results: Eighty-one patients (67\%) achieved a pCR with a median Ki-67 expression in the residual tumor of $22.5 \%$. Thirteen patients $(10.7 \%)$ relapsed and all of them belonged to the non-pCR group. In the non-relapse group, the median $\mathrm{Ki}-67$ in the residual tumor was $10 \%$ compared to $40 \%$ found in the relapse group. $(P=0.025)$. Median Ki67 post NAC was $70 \%$ in those patients who developed a central nervous disease (CNS) relapse, $40 \%$ if was a nodal or bone recurrence and $28 \%$ if it was a visceral relapse. CNS recurrence was significantly associated with higher Ki-67 levels post NAC $(P=0.010)$. Conclusions: Our results suggest that high expression levels of Ki-67 post-NAC could define different patterns of relapse in TNBC patients treated with NAC.
\end{abstract}

Keywords: Triple Negative Breast Cancer, Ki-67, Neoadjuvant Chemotherapy, Relapse, Central Nervous System

\section{Introduction}

TNBC are characterized by lack expression of estrogen receptor (ER) and progesterone receptor (PR) [1]. In addition, they lack HER2-neu gene amplification [2] and account for approximately $15-20 \%$ of all breast cancers [3]. Clinically they tend to affect women at younger age and are inclined to behave aggressively [4]. Histologically they are high-grade tumors with a high proliferation rate and have a trend to metastasize early in the liver, lung or CNS [5].
This entity is now recognized as a heterogeneous disease with a diverse molecular profile. Finding new biomarkers will eventually lead to the development of targeted therapies that may change the prognosis of this deadly neoplasm [5].

Despite being considered a chemosensitive disease, disseminated TNBC carries a dismal prognosis with a median overall survival of 18 months, which is much lower than hormone receptor positive or HER2-neu overexpressed cancers [6].

Neoadjuvant chemotherapy, initially indicated in locally advanced or inoperable cases, is now considered the standard 
of care approach for stage II and III TNBC.

Sequential anthracycline-taxane regimens lead up to $60 \%$ pCR rates $[7,8]$.

Several randomized trials have shown that the addition of CBDCA can increase the $\mathrm{pCR}$ rate [9-11].

Attaining a pCR, defined as the absence of invasive disease in the breast and axilla at the time of surgery, is considered a strong predictor of improved survival and some authors consider it could be a surrogate endpoint for event free survival [12-14].

$\mathrm{Ki}-67$ is a nuclear protein expressed in all phases of the cell cycle other than the G0. Ki-67 expression is used routinely as a proliferation marker in cancer. The monoclonal antibody MIB-1 is the most commonly used antibody for assessing Ki-67 in formalin-fixed paraffin-embedded tissue sections. The percentage of Ki-67 positive tumor cells determined by IHC is often used to stratify patients into good and poor prognostic groups [15].

In this retrospective study we analyzed a cohort of patients diagnosed with early TNBC over a 10 -year period and treated with NAC, who did not achieve a pCR; and looked at the patterns of relapse according to amount of Ki-67 expression found in the residual diseas.

\section{Materials and Methods}

\subsection{Study Population}

We retrospectively analyzed the charts of 121 TNBC patients diagnosed in our institution between 2008 and 2018 and treated with NAC and surgery.

\subsection{Inclusion of Data}

A pseudonymized database was designed for the collection of clinical-pathological information and approval was obtained from the Hospital's Reference Ethics Committee.

Tumors were staged according to the 8th edition of the AJCC TNM classification. Tumors diagnosed before the publication of this new edition were reclassified.

Every patient was initially studied with a mammogram and a breast ultrasound (US), followed by a breast difussionweighted magnetic resonance (DW-MRI). Tumors were diagnosed by image-guided thick needle biopsy (TNB) or vacuum aspiration biopsy (VAB) with US control or guided by DW-MRI when needed. The pathology report included histological grade using Nottingham Grading System [16], hormone receptor status using Allred Score [17], Ki-67 and HER2-neu expression by IHC. Uncertain IHC results were clarified employing Fluorescence In Situ Hybridization (FISH) testing.

All patients had an initial work-out which included an axillary US to detect suspicious axillary nodes, and, if so, a fine needle aspiration (FNA) was performed. A PET scan or a body $\mathrm{CT}$ and bone scan completed the diagnostic procedures to rule out distant metastasis.

Patients received chemotherapy based on taxanes and anthracyclines. Tumor response to chemotherapy was monitored with serial DW-MRI studies performed before, during and after NAC.

Patients with incomplete clinical information and/or follow-up period less than 6 months were excluded from the study.

\subsection{Surgery}

Patients underwent conservative or radical surgery, carried out by expert breast cancer surgeons. Immediate breast reconstruction was offered to patients who received radical surgery. Prophylactic contralateral mastectomy was offered to BRCA mutation carriers. Patients with a strong family history of breast cancer or with extreme anxiety concerning breast cancer diagnosis underwent prophylactic surgery. All patients were discussed at our Breast Cancer Tumor Board weekly meeting.

\subsection{Bed Tumor Localization}

For patients with no axillary involvement at initial diagnosis, localization of the residual tumor bed together with the identification of the sentinel lymph node was performed 24 hours before the planned surgery by an intratumoral injection of $148 \mathrm{MBq}$ (MegaBecquerel) of albumin $99 \mathrm{mTc}$-nanocoloid.

This procedure was carried out both for conservative or radical surgery.

Between 2008 and 2013, when a conservative surgery was performed, localization of the residual tumor bed was accomplished with a titanium harpoon, and the surgical specimen was sent for intraoperative mamographic study for margin assessment and complete harpoon extraction verification.

Between 2013 and 2018, localization was accomplished using by MRI guided ROLL/SNOLL technique (radioguided occult lesion localization) [18], checking the absence of significant activity in the surgical margins and proceeding to an intraoperative re-excision when activity was detected.

In case of no, or very low, radiotracer axillary activity, just prior to surgical incision, a periareolar subcutaneous injection of methylene blue was performed to enable visual identification of the sentinel lymph nodes.

pCR was defined as the presence of no residual invasive disease in the surgical specimen counting both (breast and axilla). We also considered a pCR case where ductal carcinoma in situ was present. Response was assessed by Miller-Payne grading system [19]. Ki-67 was assessed using MIB-1 monoclonal in formalin-fixed paraffin-embedded tissue sections. When the staining was homogenous, pathologyst counted at least three randomly selected highpower ( $\times 40$ objective) fields. When heterogeneity of Ki-67 staining was found and there was a gradient of increasing staining toward the tumor edge, those three fields were scored at the periphery of the tumor. In those cases, the invasive edge was widely considered to be the most biologically active part of the tumor and, therefore, also considered the most likely area to drive the clinical outcome 
of the disease. When different patterns of expression were found, pathologyst recorded the overall average score, counting an average of 500 and 2000 tumor cells. [15] (Figure 1).

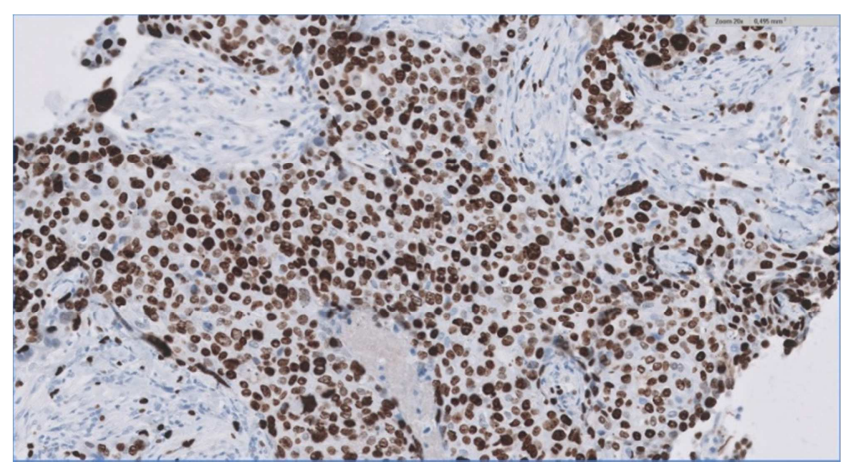

Figure 1. Ki-67 expression by immunochemistry.

Relapses were classified according to where they occurred. A loco-regional relapse was considered when the recurrence took place exclusively in the breast and/or the ipsilateral axilla. A node/bone relapse when the tumor was found in the nodes and/or bone. A visceral relapse when a metastasis was found in the liver and/or lung; and a CNS relapse when a brain and/or meningeal disease was diagnosed.

\subsection{Statistical Analysis}

A descriptive statistical analysis was performed to study patient characteristics. Frequencies were expressed as absolute numbers and percentage were used for qualitative variables. Data for quantitative variables were summarized as mean-standard deviation if they followed a normal distribution and as a median-interquartile range if not. The Kolmogorov-Smirnov test was used to check whether the data fit a normal distribution.

Pearson's chi-square test was used to compare qualitative variables, and we considered $\mathrm{P}$ values lower than 0.05 as statistically significant. SPSS software version 21 for Windows was used for data analysis (IBM Corporation, Chicago, IL, USA).

\section{Results}

Median age at diagnosis was 53 years (32-89). Fifty-two (43\%) patients were younger than 50 at diagnosis. There were only 10 patients $(8.2 \%)$ with a confirmed BRCA $1 / 2$ gene mutation.

Clinical stage at diagnosis and treatment data from all patients are depicted in Tables 1 and 2 respectively.

Table 1. Clinical stage at diagnosis of the 121 TNBC patients included in the study.

\begin{tabular}{ll}
\hline CLINICAL STAGE & N (\% OF TOTAL) \\
\hline I & $38(31.4 \%)$ \\
IIA & $51(42.1 \%)$ \\
IIB & $17(14 \%)$ \\
IIIA & $9(7.5 \%)$ \\
IIIB & $1(0.8 \%)$ \\
IIIC & $3(2.5 \%)$ \\
cT2 Nx M0 & $2(1.7 \%)$ \\
\hline
\end{tabular}

Table 2. Treatment for the 121 TNBC patients included in the study.

\begin{tabular}{lc}
\hline TREATMENT & N (\% OF TOTAL) \\
\hline Weekly paclitaxel $\left(80 \mathrm{mg} / \mathrm{m}^{2}\right)$ and carboplatin $(\mathrm{AUC} 1.5) \rightarrow \mathrm{AC}\left(60 \mathrm{mg} / \mathrm{m}^{2}-600 \mathrm{mg} / \mathrm{m}^{2}\right) \mathrm{q} 3 \mathrm{w}$ & $59(48.8 \%)$ \\
Weekly paclitaxel $\left(80 \mathrm{mg} / \mathrm{m}^{2}\right) \rightarrow \mathrm{AC}\left(60 \mathrm{mg} / \mathrm{m}^{2}-600 \mathrm{mg} / \mathrm{m}^{2}\right) \mathrm{q} 3 \mathrm{w}$ & $28(23.1 \%)$ \\
Weekly nab-paclitaxel $\left(100-125 \mathrm{mg} / \mathrm{m}^{2}\right)$ and carboplatin $(\mathrm{AUC} 1.5) \rightarrow \mathrm{AC}\left(60 \mathrm{mg} / \mathrm{m}^{2}-600 \mathrm{mg} / \mathrm{m}^{2}\right) \mathrm{q} 3 \mathrm{w}$ & $14(11.6 \%)$ \\
Keynote-522 study $(\mathrm{NCT} 03036488)$ & $10(8.3 \%)$ \\
Paclitaxel and carboplatin $\left(175 \mathrm{mg} / \mathrm{m}^{2}-\mathrm{AUC} 5\right) \mathrm{q} 3 \mathrm{w}$ & $5(4.2 \%)$ \\
Weekly paclitaxel $\left(80 \mathrm{mg} / \mathrm{m}^{2}\right)$ and carboplatin $(\mathrm{AUC} 1.5) \rightarrow \mathrm{AC} \mathrm{q} 3 \mathrm{w}\left(60 \mathrm{mg} / \mathrm{m} 2-600 \mathrm{mg} / \mathrm{m}^{2}\right)$ with bevacizumab $(15 \mathrm{mg} / \mathrm{kg}) \mathrm{q} 3 \mathrm{w}$ & $1(0.8 \%)$ \\
Weekly paclitaxel $\left(80 \mathrm{mg} / \mathrm{m}^{2}\right)$ with bevacizumab $(15 \mathrm{mg} / \mathrm{kg}) \mathrm{q} 3 \mathrm{w}$ & $1(0.8 \%)$ \\
Weekly nab-paclitaxel $\left(100-125 \mathrm{mg} / \mathrm{m}^{2}\right) \rightarrow \mathrm{AC}\left(60 \mathrm{mg} / \mathrm{m}^{2}-600 \mathrm{mg} / \mathrm{m} 2\right) \mathrm{q} 3 \mathrm{w}$ & $1(0.8 \%)$ \\
Docetaxel $100 \mathrm{mg} / \mathrm{m}^{2} \rightarrow \mathrm{AC}\left(60 \mathrm{mg} / \mathrm{m}^{2}-600 \mathrm{mg} / \mathrm{m}^{2}\right) \mathrm{q} 3 \mathrm{w}$ & $1(0.8 \%)$ \\
Weekly paclitaxel $\left(80 \mathrm{mg} / \mathrm{m}^{2}\right)$ times 12 followed by 4 cycles of Liposomal Adriamycin $\left(50 \mathrm{mg} / \mathrm{m}^{2}\right) \mathrm{q} 3 \mathrm{w}$ & $1(0.8 \%)$ \\
\hline
\end{tabular}

AC: Adriamycin-Ciclophosphamide.

Seventy-four patients $(61.2 \%)$ underwent conservative surgery and $47(38.8 \%)$ a radical procedure. Immediate reconstruction was carried out in 39 mastectomies $(83 \%)$, of which 37 (95\%) had an expander placed intraoperatively. The remaining 2 patients underwent an immediate reconstruction with autologous tissue. Contralateral prophylactic surgery was performed in $26(55.3 \%)$ patients who were eligible for radical surgery based on the recommendations of the Breast Cancer Tumor Board.

When looking at patients who underwent conservative surgery, we used ROLL/SNOLL technique in $53(71,6 \%)$ and harpoon-guided in 21 (28.4\%). Free margins were obtained in all cases.

Among patients eligible for sentinel node biopsy (SNB) ( 82 out of $121,67.8 \%$ ), we obtained a $97.6 \%$ identification rate using $99 \mathrm{Tc}$ as the single tracer in $74.4 \%$ of cases. The median sentinel nodes identified per patient were 2 [1- 4].

Our surgical complications rate was $12.3 \%$, the majority axillary seromas (7\%). None required a re-intervention.

Eighty-one patients $(67 \%)$ achieved a pCR (ypT0, ypTis) (Table 3). Nine out of the 10 patients with confirmed mutation in BRCA $1 / 2$ genes (90\%) had a pCR.

The median disease free survival (DFS) was 7.53 (1-63.4) months and median overall survival (OS) was 37.4 (1-117) 
months.

Table 3. Pathologic response after NAC in the 121 patients included in the study.

\begin{tabular}{lll}
\hline Response & N (\%) & Relapses (\%) \\
\hline pCR & $81(67 \%)$ & 0 \\
non-pCR & $40(33 \%)$ & $13(32.5 \%)$ \\
\hline
\end{tabular}

Data from relapsed patients are depicted in Table 4. Briefly, thirteen patients $(10.7 \%)$ of the whole series relapsed. All of them belonged to the non-pCR group (32.5\%) and twelve $(92.3 \%)$ had systemic disease. A patient who had initially a loco-regional recurrence, ultimately died from systemic disease 17 months later. There were no relapses in the pCR group. Seven $(58.3 \%)$ of the 12 patients with systemic disease had cerebral/meningeal metastasis (CNS relapse); 3 $(25 \%)$ with nodal and/or bone disease and $2(16.7 \%)$ with visceral metastasis. Six of the 7 patients (85.7\%) who presented with a CNS metastasis died of their disease. The median time from relapse to death was 1.9 months.

We specifically performed a Ki-67 analysis to all surgical specimens that did not achieve a pCR. The median Ki-67 in the residual tumor was $22.5 \%$. In those patients with no relapse, the median $\mathrm{Ki}-67$ in the residual tumor was $10 \%$; this median value increased to $40 \%$ in the patients that did relapse. This difference was statistically significant $(P=0.025)$ (Table 4).

In patients with a CNS relapse, the median $\mathrm{Ki}-67$ post NAC was $70 \%$, compared to $40 \%$ in the nodal/bone relapse and $28 \%$ in the visceral relapse. CNS recurrence was significantly associated significantly with a higher Ki-67 post NAC compared to non-relapsed patients $(P=0.01)$ (Table 4$)$.

Table 4. Relapses in non-pCR patients and KI-67 expression.

\begin{tabular}{|c|c|c|c|c|c|c|c|}
\hline Relapsed & N (\%) & $\begin{array}{l}\text { Median Ki-67-positive } \\
\text { cells percentage }\end{array}$ & $\begin{array}{l}\text { Systemic } \\
\text { Relapse }\end{array}$ & $\begin{array}{l}\mathrm{N}(\% \text { of } \\
\text { relapsed })\end{array}$ & Location & $\begin{array}{l}\mathrm{N}(\% \text { of systemic } \\
\text { relapse) }\end{array}$ & $\begin{array}{l}\text { Median Ki-67-positive } \\
\text { cells percentage }\end{array}$ \\
\hline $\mathrm{NO}$ & $27(67.5 \%)$ & $10 \%$ & & & & & \\
\hline \multirow{4}{*}{ YES } & \multirow{4}{*}{$13(32.5 \%)$} & \multirow{4}{*}{$40 \% *$} & NO & $1(7.7 \%)$ & & & \\
\hline & & & & & CNS & $7(58.3 \%)$ & $70 \% * *$ \\
\hline & & & YES & $12(92.3 \%)$ & Visceral & $3(25 \%)$ & $28 \%$ \\
\hline & & & & & Node/Bone & $2(16.7 \%)$ & $40 \%$ \\
\hline
\end{tabular}

* $P=0.025$ compared to non-relapsed patients

** $P=0.01$ compared to non-relapsed patients.

\section{Discussion}

TNBC accounts for approximately $15 \%$ of breast cancers. Its aggressive clinical course and lack of expression of estrogen and progesterone receptors and HER2-neu mutation, makes chemotherapy the mainstay treatment in these tumors. Although invariably lethal when diagnosed as a metastatic disease, early-stage tumors account for better prognosis and good survival rates [4].

NAC offers several advantages for the treatment of early TNBC and it is now considered the standard approach for stages II and III. Some studies have established the good prognostic value of a pathological response in these patients [12-14]. Evidence is not as clear in stage I disease, where no difference in terms of survival is found between adjuvant and this neoadjuvant approach $[20,21]$. However, there is some evidence regarding early systemic therapy in TNBC and HER2-neu positive tumors [22].

In addition, NAC gives time to screen for germline mutations that may condition the extent of surgery in these patients. This approach may also provide valuable information regarding tumor chemo-resistance in the residual disease that may help [23] design empirical trials with specific targeted therapies.

Ten patients had confirmed deleterious mutations in the BRCA $1 / 2$ genes. Nine of them $(90 \%)$ achieved a pCR. These high rates of response, despite the low numbers, are consistent with the better overall response in this subset of patients described by other groups [10].
In this single institution study involving 121 patients with TNBC treated with NAC a pCR rate of $67 \%$ was obtained, which is slightly higher than the rates reported by other groups $[7,8]$. This could be explained by the high percentage of stage I and II tumors ( $88 \%$ of the population) and the fact that almost $50 \%$ were treated with platinum-based chemotherapy.

With a median follow up of 37.4 months, none of the 81 patients that achieved a pCR have recurred. This finding supports previously published data regarding $\mathrm{pCR}$ and prognosis in this setting [12-14].

Among the 40 patients who did not achieve a pCR, 13 have recurred (32.5\%) and $12(92.3 \%)$ were systemic relapses. When we studied Ki-67 in the surgical specimen of the patients that relapsed it was significantly higher $(40 \%)$ than $\mathrm{Ki}-67$ in the residual disease of the patients who did not relapse $(10 \%, P=0.025)$. CNS relapse was especially high in our series ( 7 out of 12 systemic relapses, 58.3\%) and 6 of these patients died dramatically early, with a median time from relapse to death of 1.9 months. Interestingly, while the median $\mathrm{Ki}-67$ post NAC in the whole group of patients that relapse was $40 \%$, CNS relapse was significantly associated with a higher Ki-67 (median $70 \%, P=0.01$ ).

There are several reports in the literature describing the association between high $\mathrm{Ki}-67$ expression at diagnosis and prognosis in TNBC [24] and survival outcomes according to reduction rate of $\mathrm{Ki}-67$ between pre- and post-NAC [25-27], In our study we did not find this specific correlation.

However, regarding the prognostic value of Ki-67 
expression and the risk of a certain subtype of relapse, we found that median $\mathrm{Ki}-67$ in the residual tumor of patients that relapsed was $40 \%$, in accordance with data regarding a cutoff at $\mathrm{Ki}-67>30 \%$ as prognostic and predictive value in early stage TNBC [28]. This is especially remarkable in our patients with a SNC relapse, considering that median $\mathrm{Ki}-67$ in this group was $70 \%$. CNS progression is an aggressive and invariably lethal scenario in breast cancer and these findings, if confirmed in further studies, they could be of extraordinary importance for the design of different strategic and prophylactic approaches regarding these patients.

There are several limitations in our study, based on its retrospective character, limited number of relapses and the fact that it has been performed in a single institution.

\section{Conclusion}

The study confirms the excellent prognosis of patients with TNBC treated with neoadjuvant chemotherapy, who achieve pCR, as previously documented. Therefore, the benefit of neoadjuvant therapy as a marker of chemosensitivity is evident, so we believe that these patients should be treated in this way, even in earlier stages. Probably, the group of patients benefiting from advances in new target therapies is the one that reaches partial response. In these tumors, it seems useful to systematically determine the expression of $\mathrm{Ki}-67$ in residual disease, since its level of expression appears to be an useful marker to differentiate different patterns of relapse, and even approximate predict its appearance during the follow-up time. This value could be useful for selecting patients who require more intensive follow-up or maintenance therapy, suggesting when observing higher expression levels, to monitor possible spread to the CNS.

We think that new studies are necessary that consider the determination of the expression of $\mathrm{Ki}-67$ before and after neoadjuvant chemotherapy, with the aim to explore its definitive clinical utility in the prediction of worse prognosis of some patients with partial response. This group should be priority for clinical trials that evaluate new therapeutic strategies.

\section{Ethical Considerations}

This study is in accordance with the ethical standards of the institutional and national research committee and with the 1964 Helsinki declaration and its later amendments or comparable ethical standards.

Informed consent: this study was approved by our Ethics Committee and owing to its retrospective nature, the requirements for informed consent was waived.

\section{Conflict of Interest Statement}

The authors declare that they have no competing interests.

\section{References}

[1] Hammond ME, Hayes DF, Dowsett M, Allred DC, Hagerty KL, Badve S, et al. American Society of Clinical Oncology/College of American Pathologists guideline recommendations for immunohistochemical testing of estrogen and progesterone receptors in breast cancer. $J$ Clin Oncol. 2010; 28 (16): 2784-95.

[2] Wolff AC, Hammond MEH, Allison KH, Harvey BE, Mangu PB, Bartlett JMS, et al. Human Epidermal Growth Factor Receptor 2 Testing in Breast Cancer: American Society of Clinical Oncology/College of American Pathologists Clinical Practice Guideline Focused Update. J Clin Oncol. 2018; 36 (20): 2105-22.

[3] Perou CM, Sørlie T, Eisen MB, van de Rijn M, Jeffrey SS, Rees CA, et al. Molecular portraits of human breast tumours. Nature. 2000; 406 (6797): 747-52.

[4] Foulkes WD, Smith IE, Reis-Filho JS. Triple-negative breast cancer. N Engl J Med. 2010; 363 (20): 1938-48.

[5] Vagia E, Mahalingam D, Cristofanilli M. The Landscape of Targeted Therapies in TNBC. Cancers (Basel). 2020; 12 (4).

[6] Swain SM, Kim SB, Cortés J, Ro J, Semiglazov V, Campone $\mathrm{M}$, et al. Pertuzumab, trastuzumab, and docetaxel for HER2positive metastatic breast cancer (CLEOPATRA study): overall survival results from a randomised, double-blind, placebo-controlled, phase 3 study. Lancet Oncol. 2013; 14 (6): 461-71.

[7] Huober J, von Minckwitz G, Denkert C, Tesch H, Weiss E, Zahm DM, et al. Effect of neoadjuvant anthracycline-taxanebased chemotherapy in different biological breast cancer phenotypes: overall results from the GeparTrio study. Breast Cancer Res Treat. 2010; 124 (1): 133-40.

[8] Bagegni NA, Tao Y, Ademuyiwa FO. Clinical outcomes with neoadjuvant versus adjuvant chemotherapy for triple negative breast cancer: A report from the National Cancer Database. PLoS One. 2019; 14 (9): e0222358.

[9] Sikov WM, Berry DA, Perou CM, Singh B, Cirrincione CT, Tolaney SM, et al. Impact of the addition of carboplatin and/or bevacizumab to neoadjuvant once-per-week paclitaxel followed by dose-dense doxorubicin and cyclophosphamide on pathologic complete response rates in stage II to III triplenegative breast cancer: CALGB 40603 (Alliance). J Clin Oncol. 2015; 33 (1): 13-21.

[10] von Minckwitz G, Schneeweiss A, Loibl S, Salat C, Denkert C, Rezai M, et al. Neoadjuvant carboplatin in patients with triplenegative and HER2-positive early breast cancer (GeparSixto; GBG 66): a randomised phase 2 trial. Lancet Oncol. 2014; 15 (7): $747-56$

[11] Loibl S, O'Shaughnessy J, Untch M, Sikov WM, Rugo HS, McKee MD, et al. Addition of the PARP inhibitor veliparib plus carboplatin or carboplatin alone to standard neoadjuvant chemotherapy in triple-negative breast cancer (BrighTNess): a randomised, phase 3 trial. Lancet Oncol. 2018; 19 (4): 497509 .

[12] Cortazar P, Zhang L, Untch M, Mehta K, Costantino JP, Wolmark N, et al. Pathological complete response and longterm clinical benefit in breast cancer: the CTNeoBC pooled analysis. Lancet. 2014; 384 (9938): 164-72. 
[13] Fayanju OM, Ren Y, Thomas SM, Greenup RA, Plichta JK, Rosenberger LH, et al. The Clinical Significance of Breastonly and Node-only Pathologic Complete Response (pCR) After Neoadjuvant Chemotherapy (NACT): A Review of 20,000 Breast Cancer Patients in the National Cancer Data Base (NCDB). Ann Surg. 2018; 268 (4): 591-601.

[14] McAndrew N, DeMichele A. Neoadjuvant Chemotherapy Considerations in Triple-Negative Breast Cancer. $J$ Target Ther Cancer. 2018; 7 (1): 52-69.

[15] Dowsett M, Nielsen TO, A'Hern R, Bartlett J, Coombes RC, Cuzick J, et al. Assessment of Ki67 in breast cancer: recommendations from the International Ki67 in Breast Cancer working group. J Natl Cancer Inst. 2011; 103 (22): 1656-64.

[16] Elston CW, Ellis IO. Pathological prognostic factors in breast cancer. I. The value of histological grade in breast cancer: experience from a large study with long-term follow-up. Histopathology. 1991; 19 (5): 403-10.

[17] Phillips T, Murray G, Wakamiya K, Askaa J, Huang D, Welcher R, et al. Development of standard estrogen and progesterone receptor immunohistochemical assays for selection of patients for antihormonal therapy. Appl Immunohistochem Mol Morphol. 2007; 15 (3): 325-31.

[18] Hernández-Cortés G, Fuertes S, Martinez V, Rubio M et al. MRI guided ROLL/SNOLL in breast cancer patients treated with neoadjuvant chemotherapy. Rev Esp Med Nucl Imagen Mol. 2020. doi.org/10.1016/j.remn.2020.09.001.

[19] Ogston KN, Miller ID, Payne S, Hutcheon AW, Sarkar TK, Smith I, et al. A new histological grading system to assess response of breast cancers to primary chemotherapy: prognostic significance and survival. Breast. 2003; 12 (5): $320-7$

[20] Van der Hage JA, van de Velde CJ, Julien JP, Tubiana-Hulin M, Vandervelden C, Duchateau L. Preoperative chemotherapy in primary operable breast cancer: results from the European
Organization for Research and Treatment of Cancer trial 10902. J Clin Oncol. 2001; 19 (22): 4224-37.

[21] Wolmark N, Wang J, Mamounas E, Bryant J, Fisher B. Preoperative chemotherapy in patients with operable breast cancer: nine-year results from National Surgical Adjuvant Breast and Bowel Project B-18. J Natl Cancer Inst Monogr. 2001 (30): 96-102.

[22] Gagliato DeM, Gonzalez-Angulo AM, Lei X, Theriault RL, Giordano SH, Valero V, et al. Clinical impact of delaying initiation of adjuvant chemotherapy in patients with breast cancer. J Clin Oncol. 2014; 32 (8): 735-44.

[23] Masuda N, Lee SJ, Ohtani S, Im YH, Lee ES, Yokota I, et al. Adjuvant Capecitabine for Breast Cancer after Preoperative Chemotherapy. N Engl J Med. 2017; 376 (22): 2147-59.

[24] Wu Q, Ma G, Deng Y, Luo W, Zhao Y, Li W, et al. Prognostic Value of Ki-67 in Patients With Resected Triple-Negative Breast Cancer: A Meta-Analysis. Front Oncol. 2019; 9: 1068.

[25] Matsubara N, Mukai H, Masumoto M, Sasaki M, Naito Y, Fujii S, et al. Survival outcome and reduction rate of Ki-67 between pre- and post-neoadjuvant chemotherapy in breast cancer patients with non-pCR. Breast Cancer Res Treat. 2014; 147 (1): 95-102.

[26] Matsubara N, Mukai H, Fujii S, Wada N. Different prognostic significance of Ki-67 change between pre- and postneoadjuvant chemotherapy in various subtypes of breast cancer. Breast Cancer Res Treat. 2013; 137 (1): 203-12.

[27] Chen Ch, Zhang Y, Huang Z, Wu J, Huang W, Zhang G. Decrease in the Ki-67 index during neoadjuvant chemotherapy predicts favorable relapse-free survival in patients with locally advanced breast cancer. Cancer Biol Med. 2019. doi: 10.20892/j.issn.2095-3941.2018.0423.

[28] Zhu X, Chen L, Huang B, Wang Y, Ji L, Wu J, Di G, Liu G, Yu K, Shao Z, Wang Z. The prognostic and predictive potential of Ki-67 in triple negative breast cancer. Scientific Reports. 2020. 10: 225. doi: 10.1038/s41598-019-57094-3. 